INVESTIGACIÓN/RESEARCH

\title{
EL RETO DE CARLOS SORIA (II): CONTENIDOS E INTERACTIVIDAD
}

Juan Enrique Gonzálvez Vallés1: Universidad Camilo José Cela. España. juanen2012@gmail.com

\section{RESUMEN}

Carlos Soria revolucionó las redes sociales con un contenido casi inédito en Facebook, Twiter y Youtube. El patrocinio de BBVA otorgó al alpinista la posibilidad de dar a conocer su historia y, más allá, reflejar una serie de valores que obtuvieron una amplia y positiva respuesta en sus perfiles sociales. En esta segunda parte del estudio pormenorizado de las distintas cuentas que la entidad bancaria y el alpinista pusieron en marcha, nos centraremos en el análisis de los contenidos y la respuesta que los usuarios dieron a los mismos. Buscamos, por tanto, conocer no sólo el grado de aceptación del perfil de Carlos Soria en las redes, algo que ya afrontamos en la primera parte de este estudio, sino también hasta qué punto los usuarios aceptaron los contenidos que los perfiles propusieron $y$, de producirse una respuesta a los mismos, evaluar su trascendencia.

PALABRAS CLAVE: Carlos Soria - BBVA - Redes sociales - Patrocinio - Perfiles.

\footnotetext{
1 Autor correspondiente:

Juan Enrique Gonzálvez Vallés: Profesor del centro de estudios Formatik, asociado a la Universidad Camilo José Cela. Miembro del Grupo de Investigación Concilium de la Universidad Complutense de Madrid. Editor adjunto de las Revistas científicas SEECI y Vivat Academia. España.

Correo: juanen2012@gmail.com
} 


\title{
THE CHALLENGE OF CARLOS SORIA (II): CONTENTS AND INTERACTIVITY
}

\begin{abstract}
Carlos Soria revolutionized social networks containing almost unprecedented in Facebook, Twitter and Youtube. BBVA sponsorship gave the climber the opportunity to make known their history and beyond, reflect a set of values that were widely positive response in their social profiles. In this second part of the detailed study of the various accounts that the bank and the mountaineer launched, we will focus on the analysis of the contents and the response that users gave to them. We seek, therefore, to know not only the degree of acceptance of the profile of Carlos Soria in networks, which already face in the first part of this study, but also the extent to which users accepted the proposed content and profiles of produced a response to them, assess their significance.
\end{abstract}

KEY WORDS: Carlos Soria - BBVA - Social Networking - Sponsorship - Profiles.

\section{INTRODUCCIÓN}

Afrontamos esta segunda parte del estudio del fenómeno Carlos Soria en redes sociales, asentando nuestras bases sobre los resultados obtenidos en la primera parte. De hecho, afrontaremos desde el mismo punto de vista metodológico este nuevo estudio, puesto que creemos que una variación, ya fuera grande o pequeña, sobre éste constituiría un error claro. Podríamos obtener una desviación en los resultados y, muy probablemente, también en las conclusiones.

Sin embargo, y como no puede esperarse de otra manera al referir el título de este artículo a una segunda parte, sí que nos disponemos a variar el objeto de nuestro estudio. Si ya hemos analizado la fase inicial del patrocinio de BBVA a Carlos Soria, es decir, sus motivaciones, sus objetivos y sus articulaciones; y hemos puesto de relieve los principales resultados en cuanto a los fans, followers y suscriptores, ahora es el turno de abordar nuevas materias.

En primer lugar afrontaremos un amplio análisis de contenidos, donde pondremos el foco en el tipo de publicación y la frecuencia de publicación. Nos parecen éstas dos variables muy significativas para establecer las correspondientes conclusiones, puesto que permiten saber si tanto una como otra se están utilizando en el sentido de tratar de captar y/o fidelizar a los usuarios.

En segundo lugar, y ligado estrechamente con lo anterior, deberemos observar atentamente cuáles han sido las interacciones que se han realizado por parte de los usuarios en las diferentes publicaciones de los distintos perfiles. Podremos, de esta 
forma, establecer también conclusiones sobre cuáles son los contenidos donde más interactúan los usuarios.

Como valor añadido, y prestando especial atención a las particularidades de una red social como Facebook, también será posible analizar el nivel de "Me gusta" que recibe cada publicación. Se trata de una forma de interactuar muy particular, más sencilla y simple que cualquier tipo de comentario, y que globaliza la opinión de los usuarios. Constituirá, pues, un análisis independiente en nuestro estudio desde el punto de vista formal, pero integrado dentro del resto de análisis que establecerán las conclusiones finales.

Tendrán que ser estas conclusiones, a nuestra forma de entender, de dos tipos: por un lado, específicas de las partes del estudio que estamos llevando a cabo. Es decir, tras analizar los contenidos de los perfiles de Carlos Soria y las interacciones de los usuarios, sean éstas del tipo que sean, podremos establecer unas primeras conclusiones relacionadas con estos términos, así como lo hicimos en la primera parte de nuestro estudio.

Sin embargo, y por otro lado, también debemos establecer unas conclusiones finales que abarquen a ambas partes de nuestro estudio. No sólo daremos de esta forma cumplida finalización a este análisis sino que, de esta forma, aportaremos una visión holística al análisis de los perfiles que Carlos Soria y BBVA han llevado a cabo desde agosto de 2011.

\section{METODOLOGÍA}

En primer lugar, queremos continuar destacando que existe una sinergia significativa en el patrocinador y el patrocinado, es decir, entre BBVA y Carlos Soria. La entidad bancaria utilizo la herramienta del patrocinio, dentro de sus relaciones públicas externas, y comenzó a articular una serie de estrategias en cuanto a marketing online que son las que estamos desbrozando en este estudio.

Continuando también con la metodología de la primera arte, observaremos los datos obtenidos de la observación de los perfiles de BBVA-Carlos Soria. Tras la pertinente recogida de datos y la posterior tabulación de los mismos, es el turno ahora de obtener respuesta en forma gráfica. Así podremos obtener visiones parciales que, unidas, nos otorguen un todo gestálticamente superior.

Como decíamos, vuelve a ser nuestro método el realizar un análisis de los datos obtenido tras hacer un seguimiento detallado a los perfiles de BBVA-Carlos Soria en Facebook, Twitter y Youtube. Sin embargo, limitaremos nuestro foco a Facebook en cuanto al análisis de contenidos y la interacción de los usuarios por ser éste el perfil de mayor éxito y el que mejor ha sido explotado por parte de sus creadores. Recurriremos nuevamente a la exposición y a la demostración como el camino que más se ajusta al estudio que vamos a llevar a cabo y el que mejor nos permite establecer las conclusiones correctas. 
Pero, en este caso, focalizando sobre los contenidos publicados en los distintos perfiles y separando éstos según su tipología y especificando también la frecuencia de publicación. Aportará el estudio de estas dos variables las claves para el éxito o el fracaso de los perfiles estudiados, lo que constituye uno de los puntos más importantes de nuestro estudio.

También vamos a analizar de qué formar interactúan los usuarios con el perfil de Facebook. El análisis de los comentarios resultará muy interesante puesto que al ser abiertos y sólo con alguna restricción resultará un esfuerzo ímprobo pero a la vez gratificante. Haremos una mención especial al apartado de "Me gusta" dentro de la red social Facebook, puesto que dentro de esta categoría encontramos un elemento homogeneizador de los usuarios que va a resultar muy relevante.

Tiene que servir esta metodología para terminar de realizar un estudio holístico sobre el resultado en redes sociales del patrocinio de BBVA a Carlos Soria. Detectaremos si el proyecto es sostenible en estos niveles y, si obtenemos un resultado afirmativo, nos permitirá establecer acciones para el futuro que lo puedan seguir desarrollando. Incluso, podremos atrevernos a saber si las estrategias desarrolladas pueden ser aplicables a otros proyectos similares.

\section{ANÁLISIS Y DISCUSIÓN}

\subsection{Resultados primera parte.}

Recordamos en este punto cuáles fueron los principales hallazgos que obtuvimos en la primera parte de nuestro estudio. En primer lugar, encontramos una situación inicial en la que la imagen de los bancos estaba severamente dañada a raíz de la crisis económica y las malas prácticas bancarias de algunas entidades, que provocaron la petición de ayuda a la banca por parte de España a la Unión Europea.

Aunque BBVA no fue una de las entidades que recibió ayudas públicas también se vio lastrada por una imagen ya globalizada por parte de la opinión pública de que los bancos eran los principales responsables de la crisis, sólo por detrás del gobierno de la nación. Por ello, sus esfuerzos en relaciones públicas se intensificaron y se focalizaron en el ámbito del deporte.

Los patrocinios principales de BBVA fueron tres: la liga española, que pasó a denominarse Liga BBVA y Liga Adelante (para la primera y la segunda división); la NBA, erigiéndose el banco oficial; y Carlos Soria, el alpinista de entonces 72 años que trata de coronar las catorce montañas más altas de la tierra. Esfuerzos todo ellos que buscaban una mejora de la imagen a través de la asociación a los valores propios del deporte, en general, y de determinadas personalidades deportivas, en particular.

En este sentido, BBVA no sólo pasa a patrocinar la liga española de fútbol, sino que también adopta como embajadores mundiales de marca a dos de los máximos exponentes de esta competición: Iker Casillas, del Real Madrid; y Andrés Iniesta, del 
Barcelona. Por último, BBVA también toma la decisión de nombrar a Carlos Soria nuevo embajador mundial de marca.

Foto 1. Embajadores mundiales de marca de BBVA.
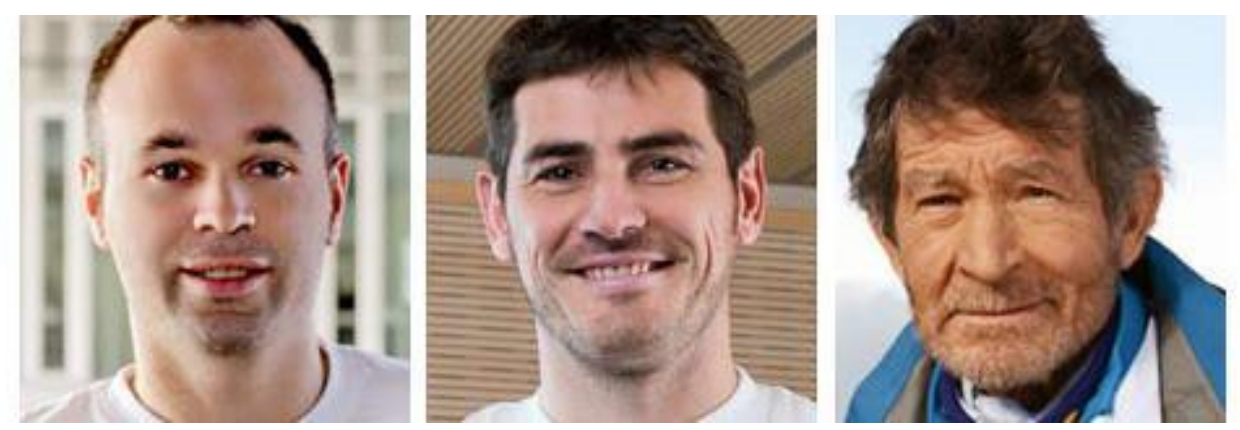

Fuente: BBVA

En el caso que nos ocupa, el del alpinista Carlos Soria, se buscaba poner un ejemplo de tenacidad, esfuerzo, superación y humildad a través del septuagenario montañero. Soria ya había conquistado once ochomiles y BBVA se comprometía a financiar sus próximas expediciones para tratar de hollar las cimas de los tres que le falta, el Annapurna, el Kanchenjunga y el Dhaulagiri.

Una apuesta que rápidamente se articuló de cara al público con la creación de tres perfiles en tres de las redes sociales más representativas: Facebook, Twitter y YouTube. Desde finales de agosto de 2011, cuando se hizo efectivo y oficial el patrocinio de BBVA a Carlos Soria, estos tres perfiles se pusieron en marcha con resultados que pronto alcanzaron la categoría de éxito casi sin precedentes, sobre todo en el caso de Facebook.

La red social que creó Mark Zuckerberg fue la que más esfuerzo recibió por parte del patrocinador, subordinando los otros perfiles a ésta primera. Fruto de esta estrategia llegaron los increíbles resultados que se registraron en cuanto a número de seguidores. Pronto se pasó de no tener ninguno a superar la extraordinaria barrera de los 200.000. Como nuestro estudio se centró en el primer año de actividad en las redes sociales, la cifra que encontramos al final de este periodo resultó asombrosa, prácticamente tocando los 233.000 fans de la página "Yo Subo con Carlos Soria". 
Gráfico 1. Histórico seguidores "Yo Subo con Carlos Soria".

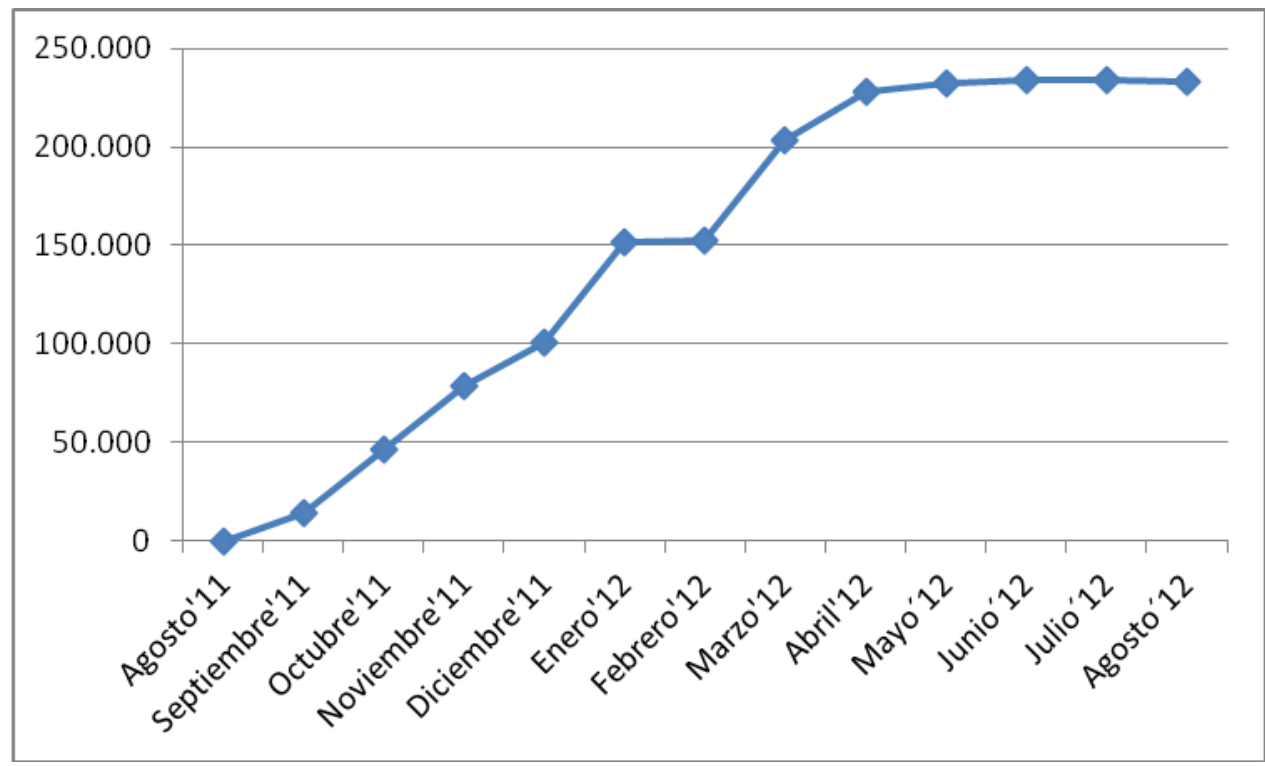

Fuente: Facebook. Elaboración propia.

Continuando con este análisis inicial de las redes sociales producidas por el patrocinio de BBVA a Carlos Soria, encontramos el caso de Twitter. Mucho menos numeroso que Facebook, los seguidores superaron por poco los 3.500 followers durante el primer año desde su creación, sobre todo porque utilizaron esta red social para tratar de dirigir al público hacia el perfil de Facebook, replicando constantemente los contenidos de éste.

\section{Gráfico 2. Histórico followers "@RetoCarlosSoria".}

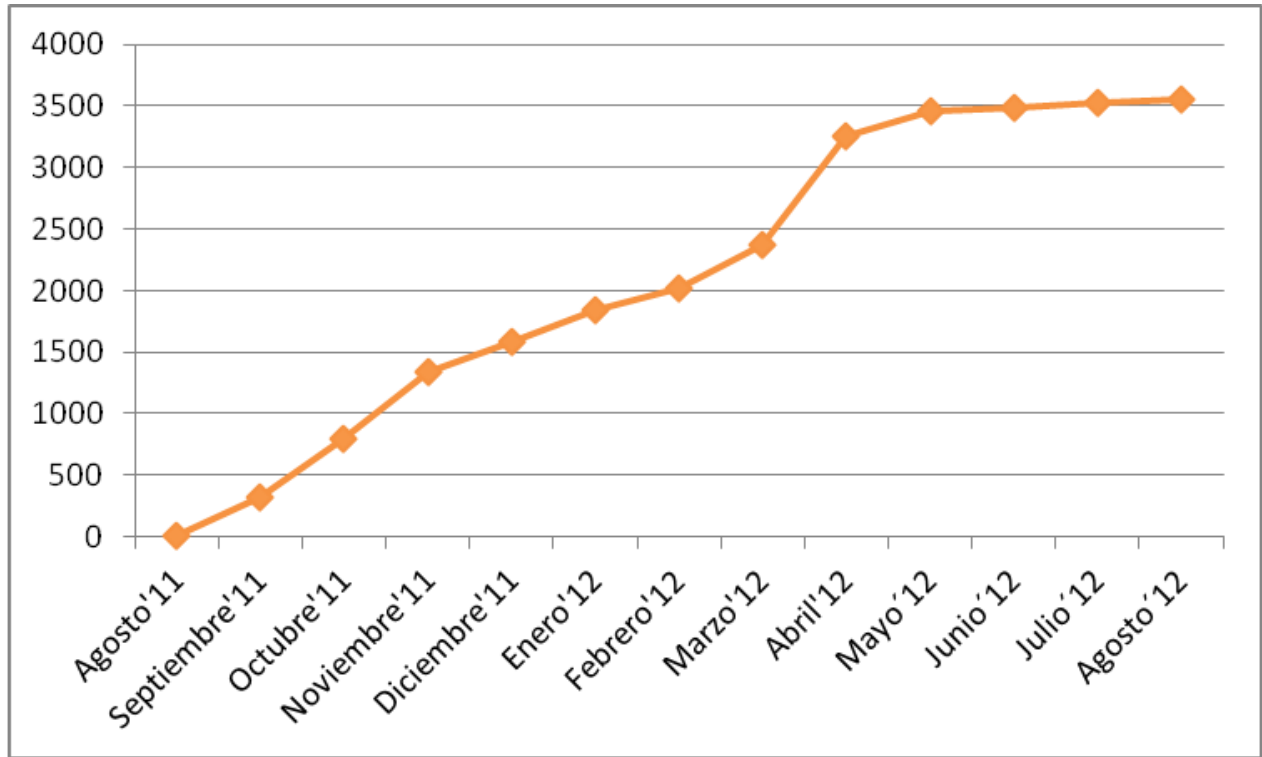

Fuente: Twitter. Elaboración propia.

Por último, también se creó un perfil en YouTube que tampoco obtuvo los esfuerzos necesarios para constituirse algo representativo. Únicamente se utilizó como 
contenedor idóneo para almacenar las piezas audiovisuales que se generaron. El resultado es que el número de suscriptores apenas se puede resultar digno de mención, puesto que ni siquiera se llegó a los 2.500 en este primer periodo de 365 días.

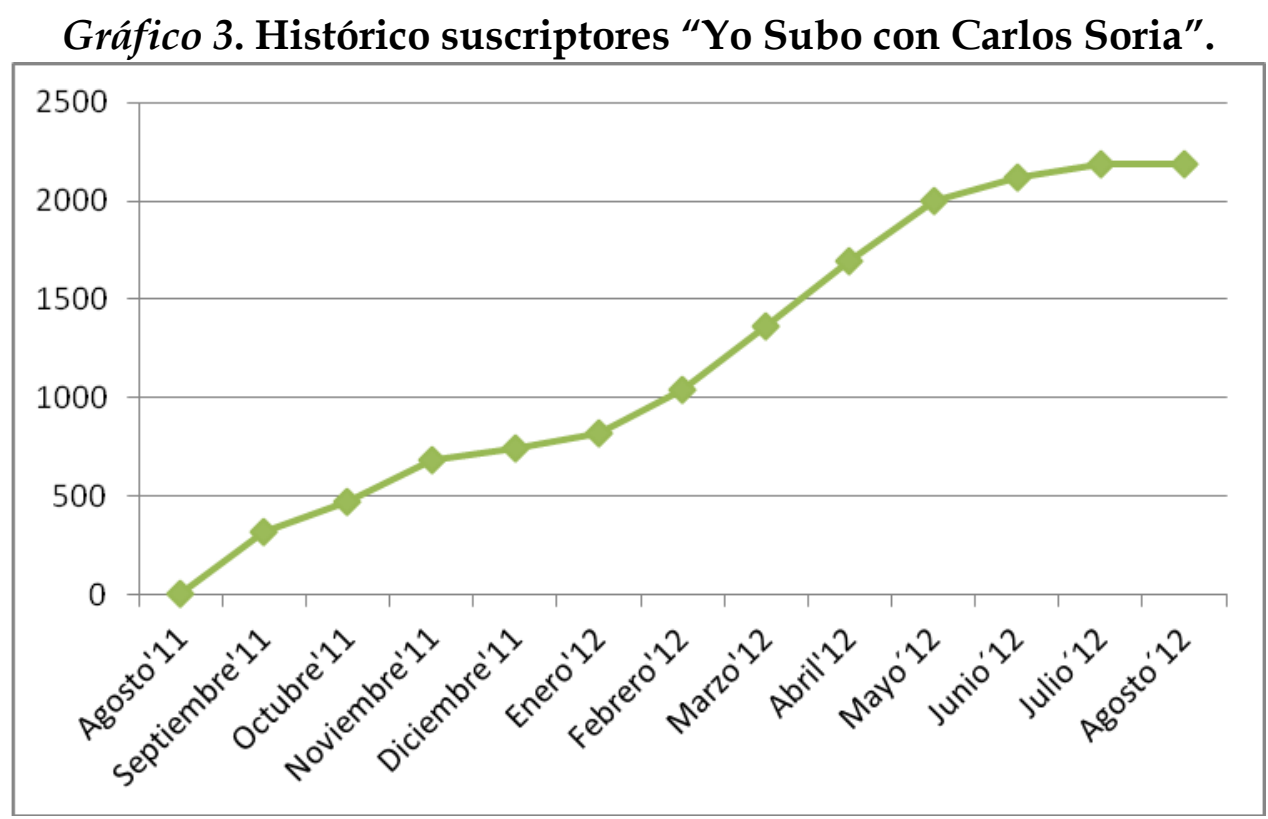

Fuente: YouTube. Elaboración propia.

A modo de novedad, aportamos ahora una comparativa entre las tres redes sociales (Facebook, Twitter y YouTube) donde, obviamente resaltan los resultados del número de usuarios, buscamos con este término una nomenclatura que aglutine, unifique o abarque a los fans, followers y suscriptores de los distintos perfiles, que 'suben' con Carlos Soria en Facebook.

Gráfico 4. Comparativa usuarios redes sociales BBVA-Carlos Soria.

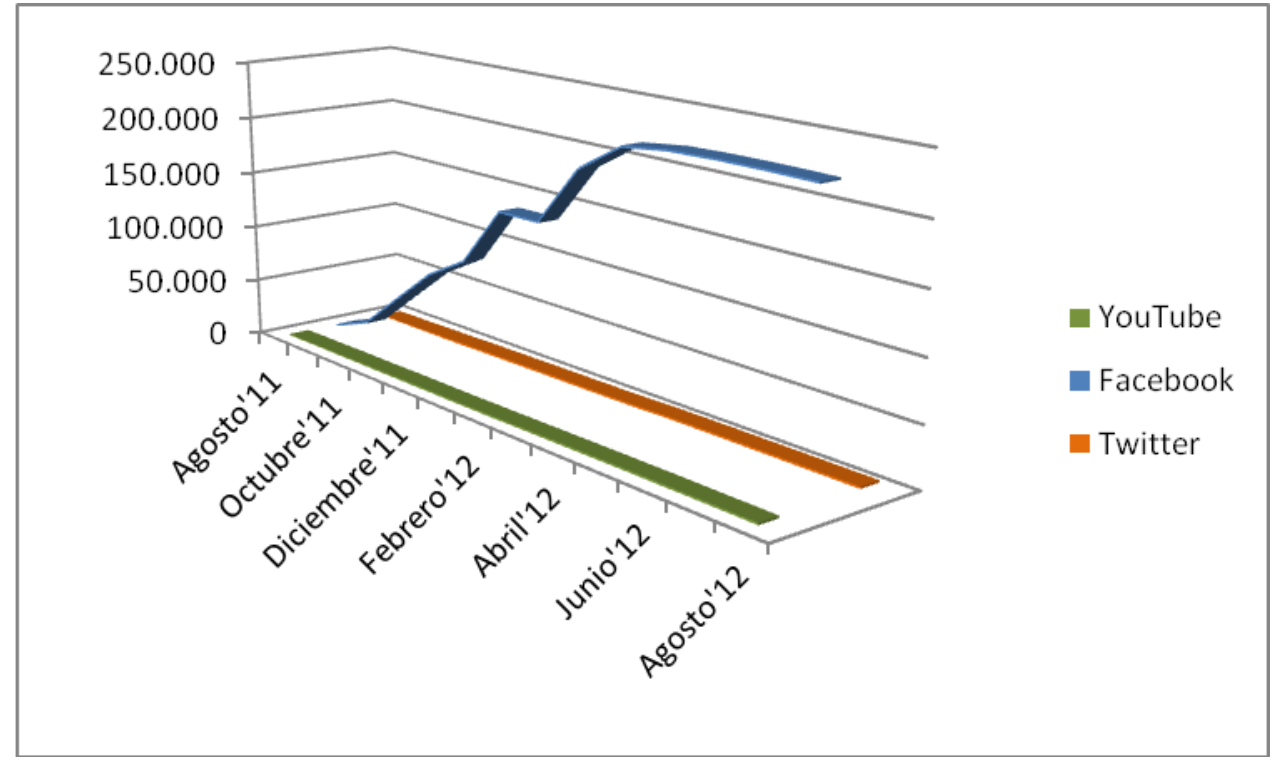

Fuente: Facebook, Twitter, YouTube. Elaboración propia. 


\subsection{Análisis de contenidos. \\ 3.2.1. Consideraciones previas.}

Establecemos en este momento los dos parámetros que consideramos más significativos a la hora de evaluar la comunicación en los perfiles de las redes sociales de BBVA-Carlos Soria: la tipología y la frecuencia. Expondremos cuáles son los diferentes tipos de contenidos que se han realizado y en qué periodos se ha publicado más y/o menos.

Sin embargo, antes de avanzar, y a tenor de los resultados obtenidos en la primera parte de nuestro estudio, debemos hacer una consideración. Hemos observado como la red social de más éxito fue sin duda Facebook, con más de 230.000 fans captados dentro de su primer año de existencia. Además, hemos podido observar como Twitter fue utilizado como un simple replicante de los contenidos de Facebook, sin aportar nada nuevo ni especial; y como YouTube se constituyó en la plataforma perfecta para aglutinar las distintas piezas audiovisuales que se realizaron.

Por todo ello, y para no entrar en análisis superfluos o incluso carentes de sentido, hemos optado por centrar nuestro foco en el perfil de Facebook, sin lugar a dudas el más paradigmático y el que nos va a permitir realizar un análisis más profuso de los contenidos. De hecho, no hay ningún contenido publicado en Twitter o YouTube que no haya sido publicado también en Facebook y, sin embargo, no todo el contenido publicado en Facebook ha sido publicado en las otras dos.

\subsubsection{Tipología.}

Debemos detallar cuáles son los tipos de publicación que se han dado dentro del perfil de Facebook "Yo Subo con Carlos Soria". En total son siete:

1. Actualización de estado: Textos sin otro tipo de elemento auditivo, visual o audiovisual.

2. Compartir: historias de otros usuarios que se comparten en el muro.

3. Enlace: una URL procedente de otra página web que se incrusta en el estado.

4. Foto: bien sea individual o pueden ser varias mediante la creación de una galería fotográfica.

5. Música: pieza sólo de audio que se inserta en el muro.

6. Pregunta: cuestiones a modo de encuesta que se publican con varias opciones de respuesta para que el usuario elija una de ellas.

7. Vídeo: también se inserta en el estado o se puede subir directamente al modo de una fotografía.

En total, se registraron 769 publicaciones en el muro de "Yo Subo con Carlos Soria", donde las fotos y los vídeos fueron los más numerosos: 
Gráfico 5. Número de publicaciones según tipología en "Yo Subo con Carlos Soria".

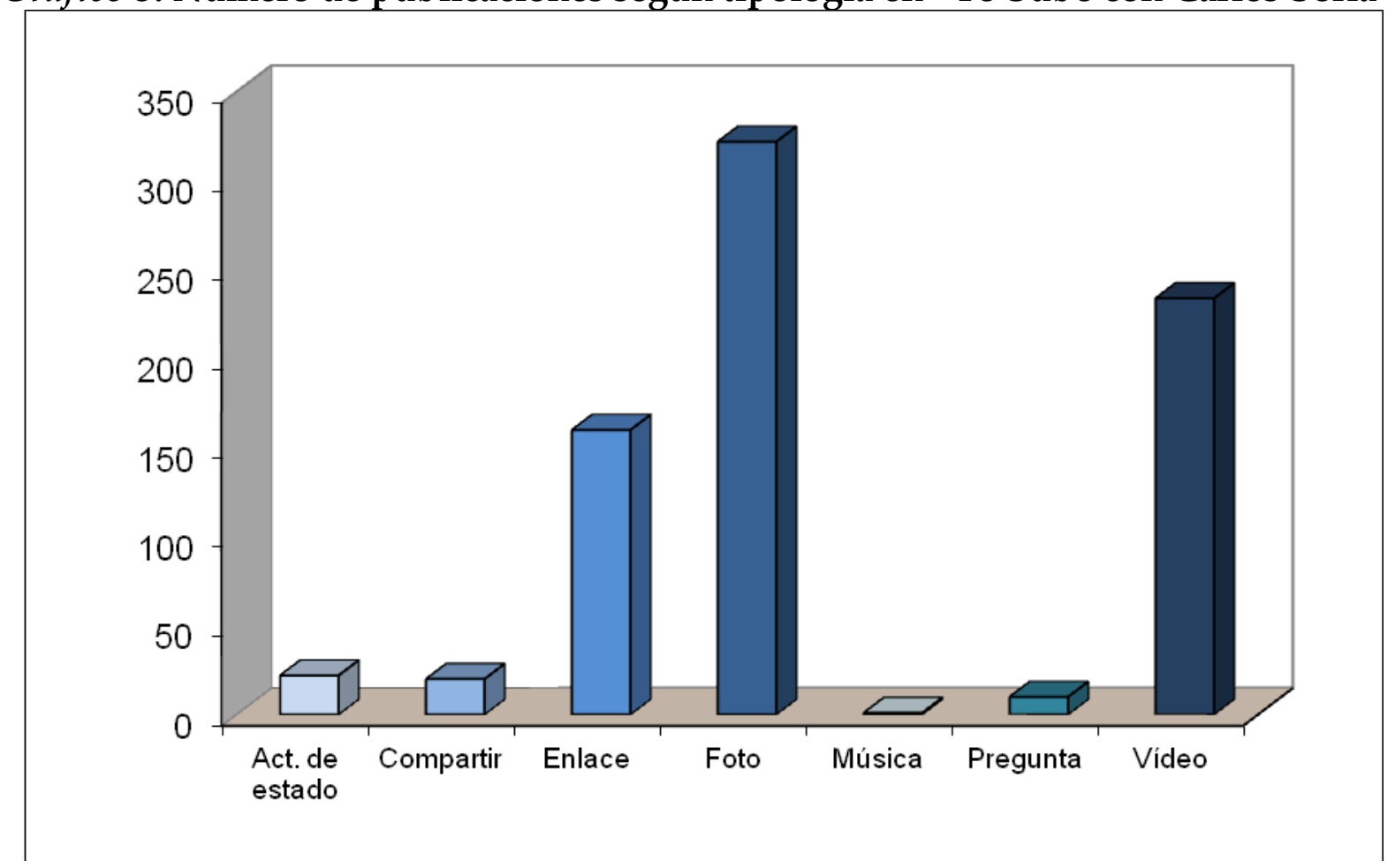

Fuente: Facebook. Elaboración propia.

A raíz de los resultados mostrados en el gráfico, observamos como los elementos audiovisuales (234) y los gráficos (322) han sido los que más veces se han reproducido en "Yo Subo con Carlos Soria". También resulta relevante observar como los enlaces externos también han poseído una gran importancia alcanzando la cifra de 160 publicaciones. Por último, casi se pueden considerar residuales el resto de publicaciones que, en el mejor de los casos, superan por poco la veintena de resultados.

\subsubsection{Frecuencia.}

Es el turno de comparar ahora este análisis con el de la frecuencia de publicación. Pretendemos obtener una visión de conjunto sobre las publicaciones puesto que, además, en el caso de Carlos Soria existen periodos bien diferenciados de mayor atención, como es el caso de las expediciones a los 'ochomiles'. Deberemos observar, por tanto, cuál es la frecuencia de las publicaciones en general y también según la tipología de las mismas. 
Gráfico 6. Número de publicaciones por meses en "Yo Subo con Carlos Soria".

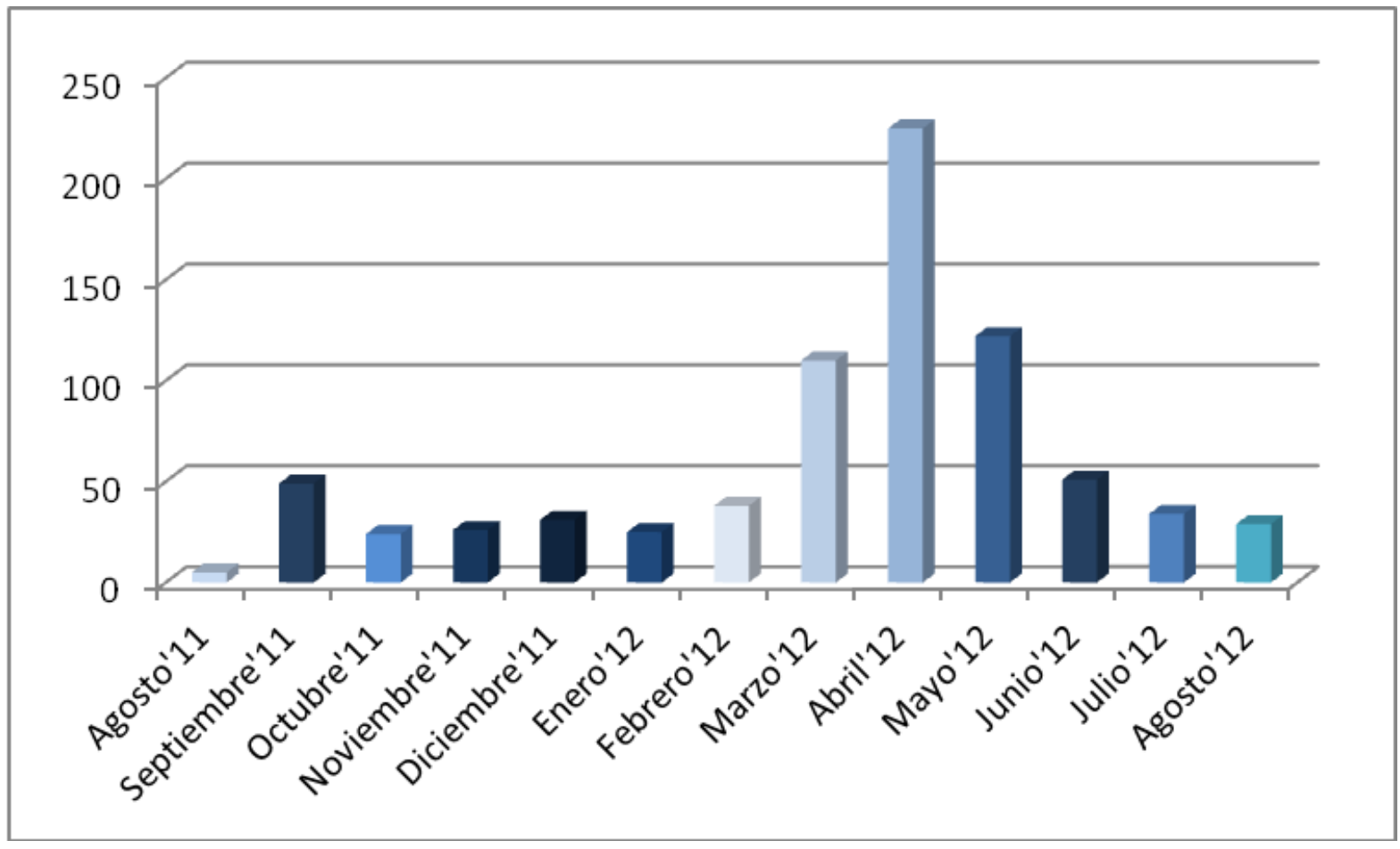

Fuente: Facebook. Elaboración propia.

Gráfico 7. Número de publicaciones por meses y según tipología.

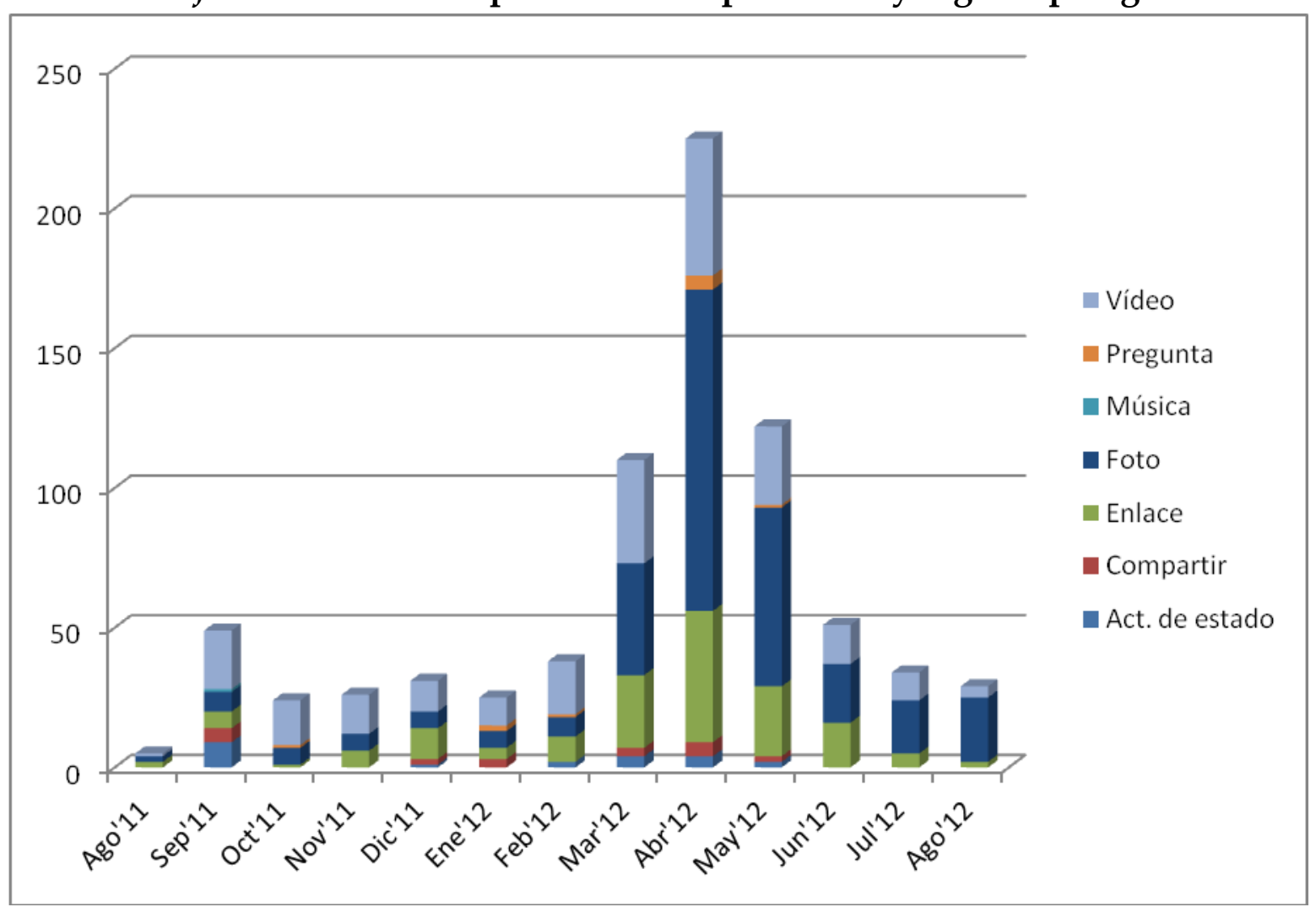

Fuente: Facebook. Elaboración propia. 
Aunque profundizaremos más en el apartado de conclusiones, apuntamos ya lo que estos gráficos denotan. En primer lugar observamos un claro pico en cuanto al número de publicaciones en el periodo correspondiente a la expedición al Annapurna, esto es, en los meses de marzo, abril y mayo de 2012. Sin embargo, y quizá por el estado embrionario del proyecto, no se produce un aumento significativo en el periodo de la expedición al Dhaulagiri, que corresponde a los meses de septiembre y octubre de 2011.

Además, constatamos el aumento de la publicación de fotografías en los periodos de expedición, tanto en la del Dhaulagiri como en la del Annapurna, aunque en ésta última el aumento es mucho más explícito. Igualmente, destacamos por el lado contrario la casi nula publicación de elementos musicales, las actualizaciones de estado y la publicación de preguntas, constituyendo elementos estadísticamente despreciables para nuestro análisis.

\subsection{Interactividad.}

Tras la observación de estos dos gráficos es obvio que nos hagamos la pregunta de si existe una correspondencia entre la tipología y la frecuencia de los contenidos publicados con el grado de interactividad de los usuarios. Pretendemos observar si este canal presenta paradojas como que un elemento con escaso número de publicaciones, como por ejemplo una pregunta, puede poseer sin embargo un alto impacto entre los fans de "Yo Subo con Carlos Soria". O si, por el contrario, existe una correlación entre los elementos más publicados, vídeos y fotos, con los 'Me gusta', los comentarios o las veces que un usuario ha compartido este contenido en su muro.

Para ello recurriremos de nuevo a tres tipos de gráficos que nos aporten una explicación global a esta hipótesis. En primer lugar, un sumatorio total de las respuestas que han recibido las publicaciones de forma mensual, con lo que obtendremos la correlación, o no, en la frecuencia de las publicaciones y su respuesta entre los usuarios. Estableceremos ahora una observación en cuanto a la categoría de pregunta, puesto que, al no recibir ni 'Me gusta' ni comentarios, asimilaremos las respuestas como si de comentarios se tratara. Es una forma que consideramos justa de medir esta interacción y que nos permite homogeneizar todo los tipos de contenidos.

En segundo lugar, observaremos también de forma gráfica cuál es la tipología de las respuestas que se han recibido de forma mensual, con lo que podremos resaltar de qué forman interactúan más los usuarios en relación con la frecuencia e intensidad de las publicaciones que se hayan realizado. Por último, debemos obtener respuesta para la relación pura entre el tipo de publicación que se haya utilizado en el perfil y la interactuación de los usuarios. 
Gráfico 8. Número de interacciones por meses en "Yo Subo con Carlos Soria".

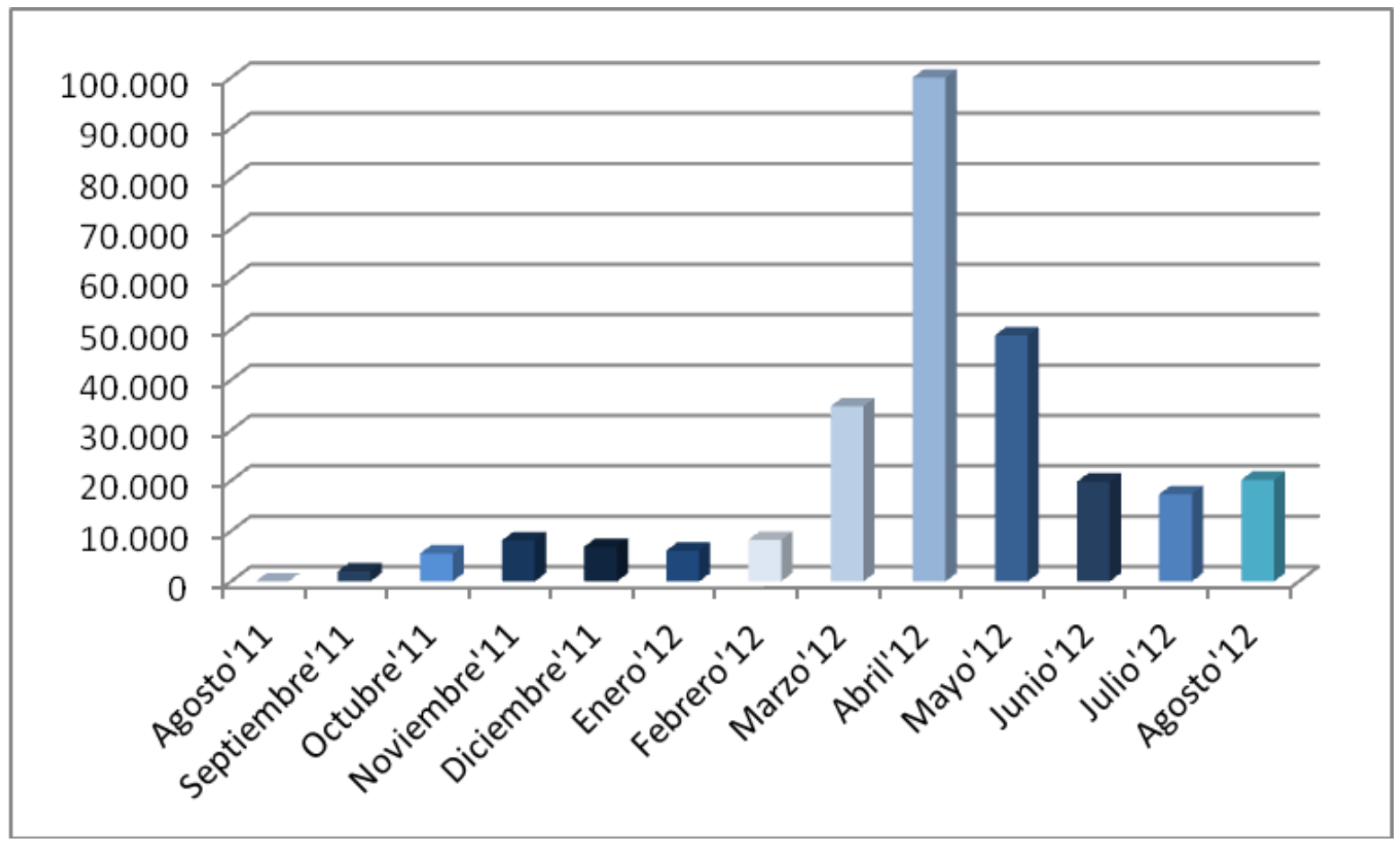

Fuente: Facebook. Elaboración propia.

Gráfico 9. Número de interacciones por meses y según tipología.

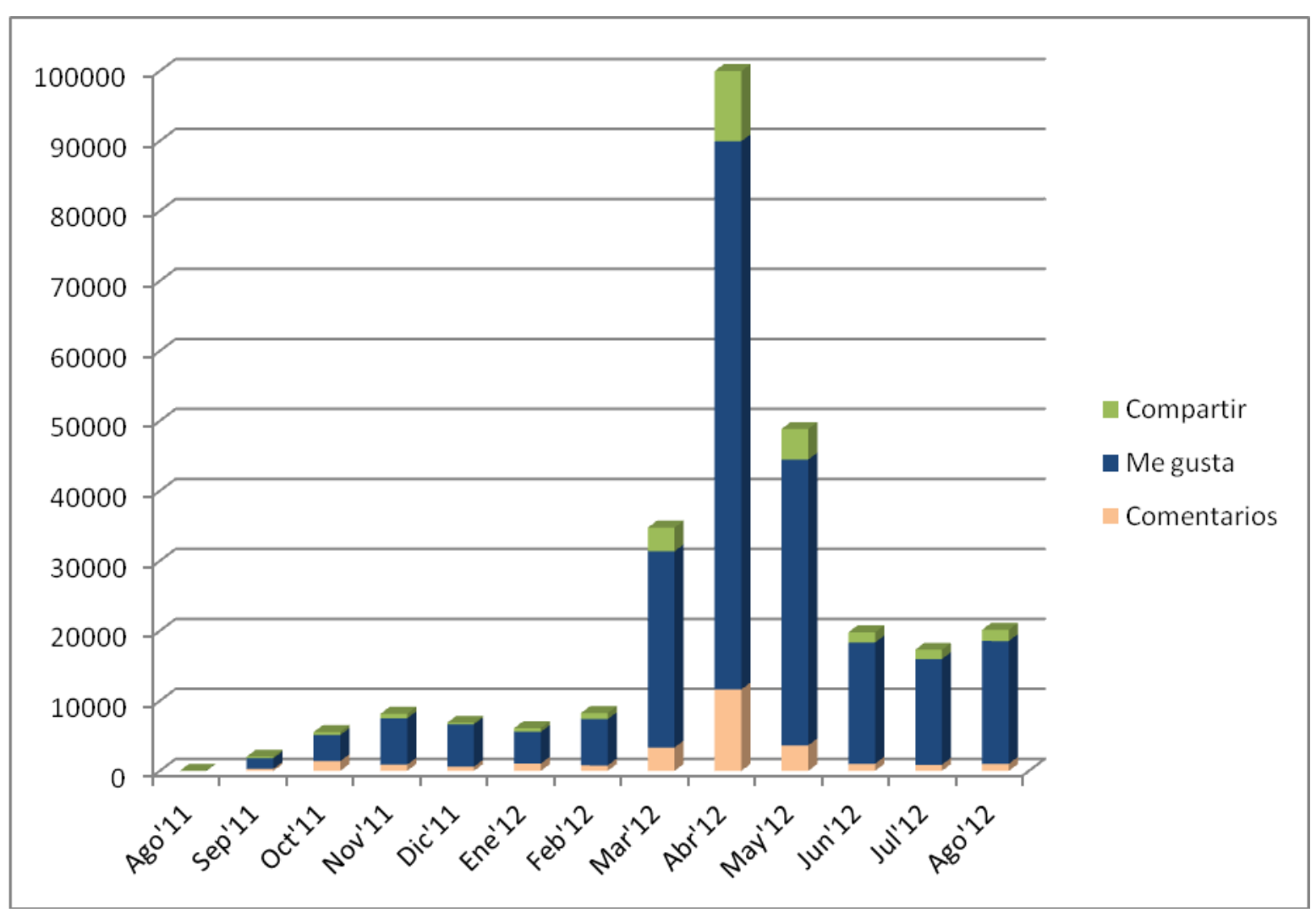

Fuente: Facebook. Elaboración propia. 
Gráfico 10. Número de interacciones por tipos y por tipo de publicación.

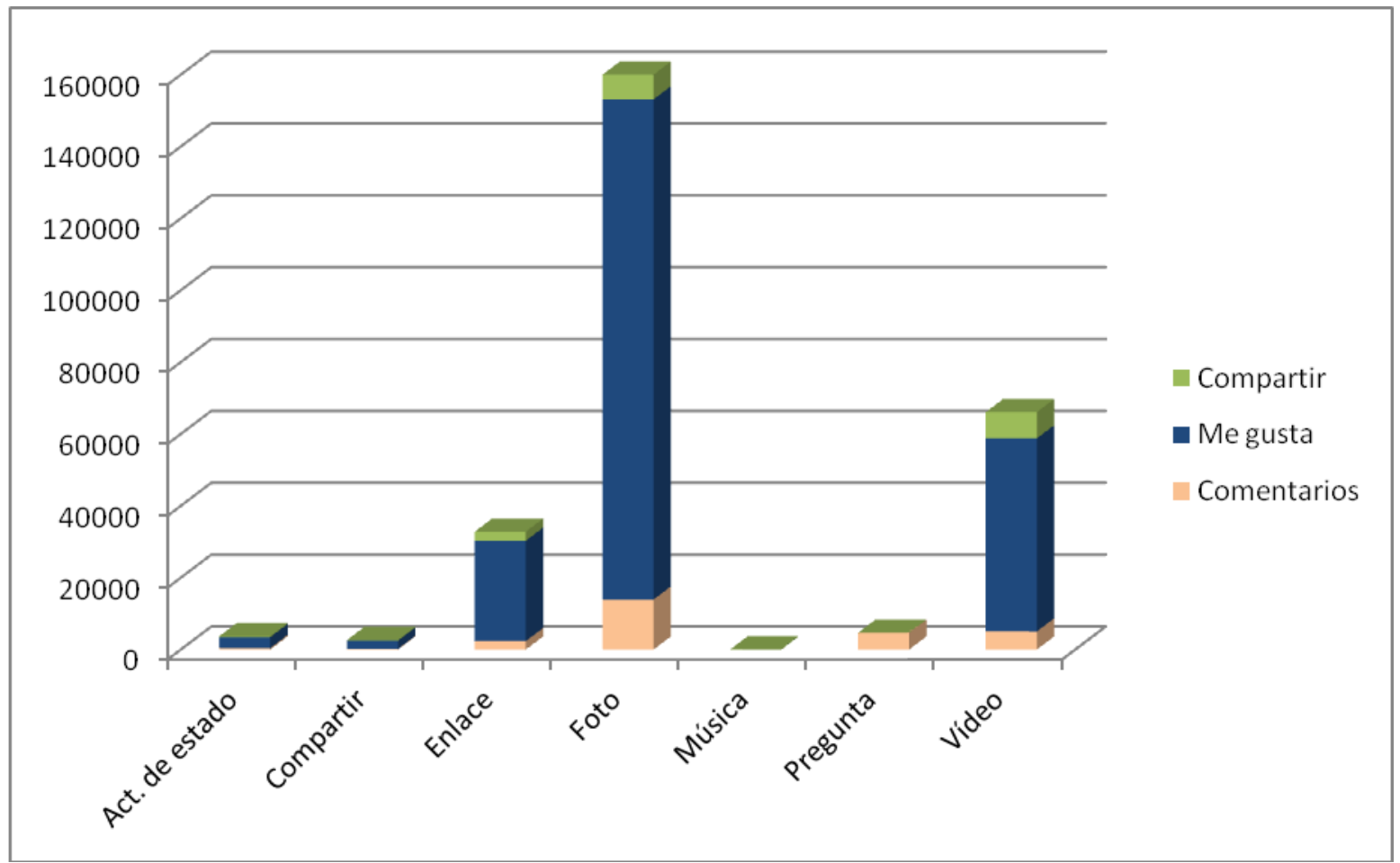

Fuente: Facebook. Elaboración propia.

Después de observar estos gráficos podemos mostrar la correlación entre las interacciones y las publicaciones del canal "Yo Subo con Carlos Soria". Primero observamos que el periodo de expedición al Annapurna fue el que, sin duda, obtuvo mayor respuesta entre los usuarios. Además, también destacan los "Me gusta" sobre el resto de interacciones posibles dentro de los contenidos. En último lugar, podemos apuntar una clara relación entre los contenidos fotográficos y el mayor grado de interacción. Son este tipo de publicaciones, seguidas de los vídeos y los enlaces, los que obtienen una mejor respuesta por parte de los fans del alpinista de 74 años en Facebook.

Apuntamos por último una interesante reflexión sobre las interacciones en "Yo Subo con Carlos Soria". Evaluamos todas ellas como positivas, es decir, que no valoran negativamente las publicaciones, ni, por supuesto, con faltas de respeto o insultos. Somos conscientes de que, como en todos los perfiles de este tipo, existe uno o varios moderadores que filtran los comentarios aparecidos en las publicaciones y purgan los que no tengan un sentido totalmente positivo. Además, los "Me gusta" son un tipo de comentario o interacción especial, cerrada y que sólo pretenden reflejar la positividad de la misma por parte del usuario.

\section{CONCLUSIONES}

A tenor de los resultados observados en nuestro estudio y su posterior análisis, y tal y como ya apuntamos en la introducción, pasaremos ahora a establecer dos tipos de conclusiones. Las primeras versarán acerca de los datos encontrados a raíz del análisis 
de la tipología de las publicaciones, su frecuencia y la interactividad de los usuarios. Además, pondremos estas en perspectiva, junto con las propias de la primera parte del estudio, para alcanzar unas conclusiones globales que cierren y completen ambos artículos.

\subsection{Específicas.}

Si atendemos a la tipología de las publicaciones del canal "Yo Subo con Carlos Soria" en Facebook, existe una clara preferencia por los elementos gráficos, fotos y galerías, y los audiovisuales, vídeos. Podemos afirmar que estos elementos, además, son más publicados durante los periodos de expedición, y exceptuamos la correspondiente al Dhaulagiri en 2011 puesto que el proyecto se encontraba una fase inicial, casi embrionaria.

También existe una clara correspondencia entre los dos elementos anteriores, tipología y frecuencia, con el grado de interactividad de los usuarios. Son muchos más los comentarios, los "Me gusta" y las veces que se ha compartido el contenido en los periodos de expediciones, que en el resto de los primeros doce meses que forman parte de nuestro estudio. Esta correlación de los tres elementos apunta al acierto de BBVA-Carlos Soria en la publicación de contenidos, siendo una de las claves de su éxito.

Además podemos extraer dos conclusiones finales sobre esta segunda parte del estudio: los "Me gusta" son, con mucha diferencia, las interacciones más usadas, seguidas por los comentarios, ya a gran distancia, y por último la posibilidad de compartir el contenido publicado en el muro del usuario. Volvemos a incidir en la particularidad del botón "Me gusta" puesto que los usuarios, en este caso los fans de Facebook, han encontrado una forma rápida y cómoda, aunque cerrada y sin posibilidad creativa alguna, de mostrar su simpatía y empatía con respecto a un contenido.

Además, hay una clara relación directa entre la tipología de los contenidos y la respuesta de los usuarios. Es cierto que las fotos y los vídeos son los tipos que reúnen un mayor número de publicaciones pero, además, son los más comentados, los más compartidos y los que más "Me gusta" acumula. En cuanto a interactividad destacamos esta relación directa entre fotos y vídeos como ejemplo más destacado y, sin embargo, también resaltamos el caso de los enlaces que obtienen un nivel de interactividad mucho menor con respecto al nivel de publicaciones, que es bastante alto. El resto de publicaciones, actualizaciones de estado, compartir, música y pregunta, vuelven a tener una relación directa entre número de publicaciones y respuesta de los usuarios, siendo bajo en ambos casos.

\subsection{Generales.}

Si afrontamos ahora las dos partes de este artículo como si fuera uno sólo podemos establecer una primera conclusión principal: los perfiles de BBVA-Carlos Soria han sido un éxito sin 
precedentes en cuanto al tipo de perfil que se afrontaba y a los resultados obtenidos. Se ha alcanzado un seguimiento extraordinario a los contenidos publicados en los perfiles, donde destacamos el esfuerzo generado en Facebook.

Precisamente ese esfuerzo ha sido el segundo gran acierto de este proyecto. Focalizar los esfuerzos hacia el perfil "Yo Subo con Carlos Soria" de Facebook ha servido para dotar al proyecto de una página de referencia y, además, de un lugar donde los usuarios han podido seguir las aventuras 'ochomilísticas' del alpinista de 74 años. Con sus más de 230.000 fans se ha erigido en referencia y ha servido para proyectar hacia los medios de comunicación el enorme éxito de este patrocinio.

En cuanto a contenidos e interacción, vemos una sinergia absoluta entre los contenidos que se publican, la respuesta a los mismos por parte de los usuarios y la fidelidad de los mismos. No sólo se captó a los fans a través de contenidos interesantes, que propiciaron un alto grado de interactividad por parte de los usuarios, sino que éstos, a través de la continuidad en el nivel de los contenidos, se han fidelizado, manteniendo la barrera de los 230.000 fans.

Aunque podría parecer lógico que, de cara a un futuro, apuntáramos a unas líneas de futuro que pusieran el esfuerzo en los otros perfiles existentes, Twitter y YouTube, nuestras recomendaciones se encaminan a una línea continuista. Si bien es cierto que la interacción puede y debe aumentarse en todos los canales, a través de menciones en Twitter y comentarios a los vídeos en YouTube, Facebook debe seguir poseyendo el foco principal en la comunicación de este patrocinio.

Esto es porque el esfuerzo ya realizado ha sido espléndido y el mantenimiento del mismo debe ser en sí un objetivo para el segundo año. El nivel de seguidores que 'suben' con Carlos Soria es tan alto que puede ser difícil de mantener si los contenidos no continúan interesando mucho a los usuarios, provocando, por tanto, un alto nivel de respuesta. El aumento de los fans parece difícil de lograrse de no darse un esfuerzo comunicativo en forma de promoción o publicidad pagada con los Facebook Ads, especialmente en los periodos de no expedición. Sin lugar a dudas, el devenir deportivo de Carlos Soria también influirá mucho en este nivel de seguimiento, puesto que una cumbre podría disparar a los seguidores del alpinista español.

\section{REFERENCIAS}

http://www.youtube.com/yosuconcarlossoria

http://www.facebook.com/yosuboconcarlossoria

http://www.twitter.com/retocarlossoria

http://prensa.bbva.com/informacion-corporativa/patrocinios/\%2825-9905\%29.html

https://ngo.bbvasuma.com/user/bbva

\section{Juan Enrique Gonzálvez Vallés}

Profesor del centro de estudios Formatik, asociado a la Universidad Camilo José Cela. Miembro del Grupo de Investigación Concilium de la Universidad Complutense de Madrid. Licenciado en Periodismo con Diploma de Estudios Avanzados. Profesionalmente ha ejercido como periodista en diversos medios de comunicaciones y entidades. 